\title{
THE HAAR MEASURE ON A COMPACT QUANTUM GROUP
}

\author{
A. VAN DAELE
}

(Communicated by Palle E. T. Jorgensen)

\begin{abstract}
Let $A$ be a $\mathrm{C}^{*}$-algebra with an identity. Consider the completed tensor product $A \bar{\otimes} A$ of $A$ with itself with respect to the minimal or the maximal $\mathrm{C}^{*}$-tensor product norm. Assume that $\Delta: A \rightarrow A \bar{\otimes} A$ is a non-zero *-homomorphism such that $(\Delta \otimes l) \Delta=(l \otimes \Delta) \Delta$ where $l$ is the identity map. Then $\Delta$ is called a comultiplication on $A$. The pair $(A, \Delta)$ can be thought of as a 'compact quantum semi-group'.

A left invariant Haar measure on the pair $(A, \Delta)$ is a state $\varphi$ on $A$ such that $(l \otimes \varphi) \Delta(a)=\varphi(a) 1$ for all $a \in A$. We show in this paper that a left invariant Haar measure exists if the set $\Delta(A)(A \otimes 1)$ is dense in $A \bar{\otimes} A$. It is not hard to see that, if also $\Delta(A)(1 \otimes A)$ is dense, this Haar measure is unique and also right invariant in the sense that $(\varphi \otimes \imath) \Delta(a)=\varphi(a) 1$.

The existence of a Haar measure when these two sets are dense was first proved by Woronowicz under the extra assumption that $A$ has a faithful state (in particular when $A$ is separable).
\end{abstract}

\section{INTRODUCTION}

Let $A$ be a $\mathrm{C}^{*}$-algebra. Consider the completion $A \bar{\otimes} A$ of the tensor product of $A$ with itself with respect to the minimal or the maximal $\mathrm{C}^{*}$-tensor product norm. A *-homomorphism $\Delta$ of $A$ into the multiplier algebra $M(A \bar{\otimes} A)$ of $A \bar{\otimes} A$ is called a comultiplication if it is non-degenerate (that is, if $\Delta(A)(A \otimes A)$ is dense in $A \bar{\otimes} A)$ and if it satisfies the coassociativity rule $(\Delta \otimes l) \Delta=(l \otimes \Delta) \Delta$, where $l$ denotes the identity map. Remark that the maps $\Delta \otimes l$ and $l \otimes \Delta$ have unique extensions from $A \otimes A$ to $A \bar{\otimes} A$ by continuity and to $M(A \bar{\otimes} A)$ because $\Delta$ is non-degenerate.

If $A$ is abelian, then $A$ can be identified with the $\mathrm{C}^{*}$-algebra $C_{0}(G)$ of continuous complex functions tending to 0 at infinity on a locally compact space $G$. We can identify $A \bar{\otimes} A$ with $C_{0}(G \times G)$ and $M(A \bar{\otimes} A)$ with the $\mathrm{C}^{*}$-algebra of bounded continuous complex functions on $G \times G$. The comultiplication $\Delta$ defines a multiplication on $G$ by $f(s t)=(\Delta f)(s, t)$ because $\Delta$ is non-degenerate. The multiplication is associative because $\Delta$ is coassociative.

Therefore, when $A$ is any $\mathrm{C}^{*}$-algebra, we think of a pair $(A, \Delta)$ as a 'locally compact quantum semi-group'. In general, it is not yet clear what the extra conditions on $\Delta$ must be in order to have a 'locally compact quantum group'. These conditions must be such that a nice theory can be developed along the

Received by the editors March 28, 1994.

1991 Mathematics Subject Classification. Primary 46L05; Secondary 22C05, 16W30. 
lines of the theory of locally compact groups. The crucial point is the existence and uniqueness of (the equivalent of) the Haar measure.

In recent years, progress has been made on this project. First of all, there is the important work of Woronowicz on the compact case ([6] and [7]). Recently, also the discrete case has been studied (see [1], [4] and [5]). The finitedimensional case is also well understood [3].

In all cases, the density of the spaces $\Delta(A)(A \otimes 1)$ and $\Delta(A)(1 \otimes A)$ in $A \bar{\otimes} A$ appears as a natural condition. This is no surprise; in the case $A=C_{0}(G)$, this condition is equivalent to the cancellation property in $G$. Furthermore, it is known that a compact semi-group with cancellation is a group [2]. And $G$ is compact if and only if $A$ has an identity.

As it turns out, if $(A, \Delta)$ is a pair of a $C^{*}$-algebra $A$ with an identity and a comultiplication $\Delta$ satisfying the above density conditions, then there exists a unique Haar measure, that is, a state $\varphi$ on $A$ such that $(\varphi \otimes l) \Delta(a)=$ $(l \otimes \varphi) \Delta(a)=\varphi(a) 1$ for all $a \in A$. Although there are cases where this Haar measure is not faithful, enough properties can be proven so that the pair $(A, \Delta)$ deserves the name 'compact quantum group'.

The theory of compact quantum groups was developed by Woronowicz in [6] and [7] where he also proves the existence of the Haar measure if $A$ admits a faithful state. In this note, we show that this condition is not necessary. In fact, we prove a result that is slightly more general. We show that there is a left Haar measure if the set $\Delta(A)(A \otimes 1)$ is dense in $A \bar{\otimes} A$. It is not necessarily unique. Of course, it will be unique and also right invariant if also $\Delta(A)(1 \otimes A)$ is dense. It should be mentioned that the proof basically uses the techniques that also were used by Woronowicz.

\section{THE MAIN RESULT}

Let $A$ be a $C^{*}$-algebra with identity. Let $\Delta$ be a comultiplication on $A$. We will use the product of bounded linear functionals on $A$ defined by

$$
\left(w_{1} w_{2}\right)(a)=\left(w_{1} \otimes w_{2}\right) \Delta(a)
$$

when $a \in A$ and $w_{1}, w_{2} \in A^{*}$.

2.1 Lemma. Let $\omega$ be a state on $A$. Then there is a state $\varphi$ such that $\varphi \omega=$ $\omega \varphi=\varphi$.

Proof. Define

$$
\omega_{n}=\frac{1}{n}\left(\omega+\omega^{2}+\cdots+\omega^{n}\right) .
$$

Then $\omega_{n}$ is a state for all $n$. Consider a weak *-limit point $\varphi$ of the sequence $\left(\omega_{n}\right)$. It is still a state because $A$ has an identity. As

$$
\left\|\omega_{n} \omega-\omega_{n}\right\|=\frac{1}{n}\left\|\omega^{n+1}-\omega\right\| \leq \frac{2}{n},
$$

we get in the limit $\varphi \omega=\varphi$. Similarly $\omega \varphi=\varphi$.

The above argument is standard; see e.g. [6] and [7]. Also for the following result, we use the techniques of [6] and [7]. 
2.2 Lemma. Assume that $\Delta(A)(A \otimes 1)$ is dense in $A \bar{\otimes} A$. Let $\omega$ and $\varphi$ be states such that $\omega \varphi=\varphi$. If $\rho \in A^{*}$ and $0 \leq \rho \leq \omega$, then also $\rho \varphi=\rho(1) \varphi$.

Proof. Let $a \in A$ and define $b=(l \otimes \varphi) \Delta(a)$, where again $l$ denotes the identity map on $A$. Then

$$
(l \otimes \omega) \Delta(b)=(l \otimes \omega \varphi) \Delta(a)=(l \otimes \varphi) \Delta(a)=b .
$$

So

$$
\begin{aligned}
(l \otimes & \omega)\left((\Delta(b)-b \otimes 1)^{*}(\Delta(b)-b \otimes 1)\right) \\
& =(l \otimes \omega) \Delta\left(b^{*} b\right)+b^{*} b-(l \otimes \omega)\left(\Delta\left(b^{*}\right)(b \otimes 1)\right)-(l \otimes \omega)\left(\left(b^{*} \otimes 1\right) \Delta(b)\right) \\
& =(l \otimes \omega) \Delta\left(b^{*} b\right)-b^{*} b .
\end{aligned}
$$

Now, take any state $\psi$ such that $\psi \omega=\psi$. Such a state exists by Lemma 2.1. If we apply $\psi$ to the above equation we will get

$$
(\psi \otimes \omega)\left((\Delta(b)-b \otimes 1)^{*}(\Delta(b)-b \otimes 1)\right)=0 .
$$

By the Cauchy-Schwartz inequality we get

$$
(\psi \otimes \omega)((c \otimes d)(\Delta(b)-b \otimes 1))=0
$$

for all $c, d \in A$. If we now use that $b=(l \otimes \varphi) \Delta(a)$, we get

$(\psi \otimes \omega \otimes \varphi)((c \otimes d \otimes 1)(\Delta \otimes l) \Delta(a))=\omega(d)(\psi \otimes \varphi)((c \otimes 1) \Delta(a))$.

Now

$$
\begin{aligned}
(c \otimes d \otimes 1)(\Delta \otimes l) \Delta(a) & =(c \otimes d \otimes 1)(l \otimes \Delta) \Delta(a) \\
& =(1 \otimes d \otimes 1)(l \otimes \Delta)((c \otimes 1) \Delta(a)) .
\end{aligned}
$$

By assumption, linear combinations of elements of the form $(c \otimes 1) \Delta(a)$ are dense in $A \bar{\otimes} A$. So we can, in the above equality, replace $(c \otimes 1) \Delta(a)$ by $1 \otimes q$. Then we obtain

$(\psi \otimes \omega \otimes \varphi)((1 \otimes d \otimes 1)(l \otimes \Delta)(1 \otimes q))=\omega(d)(\psi \otimes \varphi)(1 \otimes q)$

and so, because $\psi(1)=1$, we get

$$
(\omega \otimes \varphi)(d \otimes 1) \Delta(q)=\omega(d) \varphi(q)
$$

for all $d, q \in A$.

Now consider the G.N.S. construction for $\omega$. Let $\xi_{0}$ be the cyclic vector and $\pi$ the representation. Denote $r=(l \otimes \varphi) \Delta(q)$. Then we find

$$
\left\langle\pi(r) \xi_{0}, \pi\left(d^{*}\right) \xi_{0}\right\rangle=\varphi(q)\left\langle\xi_{0}, \pi\left(d^{*}\right) \xi_{0}\right\rangle
$$

Because this is true for all $d$, we get

$$
\left\langle\pi(r) \xi_{0}, \xi\right\rangle=\varphi(q)\left\langle\xi_{0}, \xi\right\rangle
$$

for all vectors $\xi$. Now, because $0 \leq \rho \leq \omega$, there is a vector $\xi$ so that $\rho(x)=\left\langle\pi(x) \xi_{0}, \xi\right\rangle$ for all $x$. Hence $\rho(r)=\varphi(q) \rho(1)$. But $\rho(r)=(\rho \varphi)(q)$. And because this is true for all $q$, we get $\rho \varphi=\rho(1) \varphi$. This completes the proof.

The main result follows easily by using a compactness argument. 
2.3 Theorem. If $A$ is a $\mathrm{C}^{*}$-algebra with identity and $\Delta$ a comultiplication on $A$ such that $\Delta(A)(A \otimes 1)$ is dense in $A \bar{\otimes} A$, then there is a state $\varphi$ so that $\rho \varphi=\rho(1) \varphi$ for all $\rho \in A^{*}$.

Proof. For any $\omega \geq 0$, define

$$
K_{\omega}=\left\{\varphi \in A^{*} \mid \varphi \text { is a state and } \omega \varphi=\omega(1) \varphi\right\} .
$$

This is a compact subset of $A^{*}$ for the weak *-topology. It is non-empty because of Lemma 2.1. By Lemma 2.2 we have that $K_{\omega} \subseteq K_{\rho}$ when $0 \leq \rho \leq \omega$. It follows that $K_{\omega_{1}+\omega_{2}} \subseteq K_{\omega_{1}} \cap K_{\omega_{2}}$ for any pair of positive linear functionals $\omega_{1}, \omega_{2}$. Therefore the intersection of all $K_{\omega}$ is non-empty. This proves the result.

If $A$ is a $\mathrm{C}^{*}$-algebra with identity and $\Delta(a)=1 \otimes a$ for all $a$, then $\Delta$ is a comultiplication and $\Delta(A)(A \otimes 1)$ is dense in $A \bar{\otimes} A$ trivially. In this case, every state is a left Haar measure. In the abelian case, this corresponds to the semigroup with the product $s t=t$ for all $s, t$. In this case of course, $\Delta(A)(1 \otimes A)$ is no longer dense (except when $A$ is trivial).

If also this set is dense, we easily get the following.

2.4 Theorem. If $A$ is a $C^{*}$-algebra with identity and $\Delta$ a comultiplication such that $\Delta(A)(A \otimes 1)$ and $\Delta(A)(1 \otimes A)$ are dense in $A \bar{\otimes} A$, then there is a unique state $\varphi$ so that $\rho \varphi=\varphi \rho=\rho(1) \varphi$ for all $\rho \in A^{*}$.

Proof. By the previous theorem we know that there is a state $\varphi$ such that $\rho \varphi=\rho(1) \varphi$ for all $\rho \in A^{*}$. Similarly, or by applying this theorem to the opposite comultiplication $\Delta^{\prime}$, obtained from $\Delta$ using the flip, there is a state $\psi$ such that $\psi \rho=\rho(1) \psi$ for all $\rho \in A^{*}$. Then $\psi \varphi=\varphi$ by the first formula and $\psi \varphi=\psi$ by the second formula. So $\psi=\varphi$ and the result is proved.

\section{ACKNOWLEDGMENTS}

We would like to thank Professor A. Lazar for his hospitality while we were visiting the University of Tel Aviv. Without the many interesting discussions we had with him, this note would not have been written. We also are grateful to Professor M. Rieffel for his hospitality at the University of California in Berkeley where we revised this note.

\section{REFERENCES}

1. E. G. Effros and Z.-J. Ruan, Discrete quantum groups I. The Haar measure, preprirt, UCLA, 1993.

2. K. H. Hofmann, Elements of compact semi-groups, Merrill, Columbus, OH, 1966.

3. A. Van Daele, The Haar measure on finite quantum groups, preprint, K. U. Leuven, 1992.

4. __ Quasi-discrete locally compact quantum groups, preprint, K. U. Leuven, 1993.

5. __ Discrete quantum groups, preprint, K. U. Leuven, 1993.

6. S. L. Woronowicz, Compact matrix pseudo-groups, Comm. Math. Phys. 111 (1987), 613-665.

7. _ Compact quantum groups, preprint, University of Warsaw, 1992.

Department of Mathematics, Katholieke Universiteit Leuven, CelestijnenlaAn 200B, B-3001 HeVerlee, Belgium

E-mail address: Alfons.VanDaele@iis.kuleuven.ac.be 